\title{
Long-term cross-sex hormone treatment is safe in transsexual subjects
}

\author{
Maria Cristina Meriggiola and Marta Berra \\ Asian Journal of Andrology (2012) 14, 813-814; doi:10.1038/aja.2012.89; Published online: 27 August 2012
}

$\mathrm{T}$ he European Journal of Endocrinology recently published a paper (Asscheman et al.) presenting mortality data from more than a thousand transsexuals followed for a median time of 18 years and who had undergone previous or were currently on long term cross-sex hormonal treatment. Transsexualism is a rare condition and in scientific literature there are few reports on the long-term safety of different treatment protocols and on the physical and psychological outcomes of medical treatments. The safety of long-term high doses oestrogen or testosterone in subjects of the opposite sex has been debated and data on long term effects are scant at best. The Asscherman paper therefore represents a very important and reassuring reference for professionals working in this field suggesting that mortality is increased among transsexuals although due to causes unrelated to cross sex replacement therapy. These results will benefit the care and treatment of these subjects.

In April 2011, the European Journal of Endocrinology published an important paper in scientific literature dealing with transsexual subjects. ${ }^{1}$ The authors presented mortality data on 966 male-to-female $(\mathrm{MtF})$ and 365 female-to-male (FtM) transsexual subjects who had undergone long-term standard cross-sex hormonal treatment. ${ }^{2}$ They concluded that in MtF subjects, mortality increases when compared to the general male and female population. The study showed that this increased mortality is mainly due to non-hormonal causes; suicide, AIDS and illicit drug related deaths were higher when compared to the general population.

Center for Protection of Sexual Health, Physiopathology of Reproduction and Gynecology, University of Bologna and S. Orsola-Malpighi Hospital, Bologna 40138, Italy Correspondence: Dr MC Meriggiola

(cristina.meriggiola@unibo.it)
Transsexualism is a rare condition affecting approximately one in 12000 males and one in 30000 females $^{3}$ and collecting data from more than 1000 patients treated within the same medical center is remarkable. Compared to other papers dealing with this topic, this article appears particularly relevant considering both the sample size and the length of follow-up. Good data on mortality rates in transsexual subjects undergoing cross-sex hormone treatment with or without SRS are scanty. Asscheman and colleagues ${ }^{4,5}$ are not new to this research having reported the effects of hormonal treatment in 1989 and 1997 and, since then, findings from this group have confirmed that increased mortality in these subjects is not due to hormonal causes. The length of follow-up is also remarkable since it was suggested in a previous report that mortality rate differences start to appear after 10 years. In this paper, over $90 \%$ of the subjects were followed for more than 10 years with a median time of 18 years.

Previous studies on transsexuals have documented an increased incidence of thromboembolism in $\mathrm{MtF}$ using ethinyl estradiol (EE), $6 \%-8 \%$ of whom had a vascular event, not confirmed when other oestrogen preparations are used. ${ }^{3}$ Other factors such as age, smoking and carriage of genetic polymorphism are factors known to play a fundamental role. ${ }^{6-12}$ The current recommendation is therefore to avoid the use of $\mathrm{EE}$ and to prefer natural or conjugated estrogens particularly for subjects over 40 years of age. ${ }^{13}$ This paper reinforces this recommendation showing that the risk of cardiovascular events is increased threefold in subjects who did not want to stop EE when compared to past or non-users. The good news is that once EE is stopped, the risk seems to return to being indifferent from control subjects. This paper reinforce this recommendation showing that current risk of cardiovascular events are threefold increased in those subjects that did not want to stop EE compared to past or never users. It remains to be established whether it is cost-effective to screen $\mathrm{MtF}$ before or during hormonal treatment for thrombophilia because genetic predisposition may further increase the risk.

Another interesting aspect emerging from the Asscheman paper is the difference in the mortality rate between FtM and MtF transsexuals. FtM subjects do not present a higher mortality rate nor does cause-specific mortality appear to be significantly different to that of the general population. Even considering that FtM are numerically inferior compared to $\mathrm{MtF}$ and that FtM subjects aged over 65 years are small number, still this is a significant difference between the two groups. Testosterone treatment is safe in these women and does not lead to increased cardiovascular mortality. ${ }^{14,15}$ The fact that testosterone has apparent detrimental effects on surrogate markers of cardiovascular risk ${ }^{16}$ but does not increase cardiovascular mortality is, in our opinion, intriguing and certainly calls for wider investigation on the way in which testosterone works in women. ${ }^{17}$

Some other important issues remain open and also require further research. Of extreme interest is the evaluation of the role of sex reassignment surgery and whether it has a real 'therapeutic' effect on the relief of dysphoria. ${ }^{18,19}$ Scientific literature is not univocal on this point: psychological evaluation reports agree that reassignment surgery improves quality of life, but apparently it does not cure all aspects of gender dysphoria. In some transsexual groups, the suicide rate appears to be higher in subjects who did not undergo surgery, but other studies do not confirm these findings. The Dutch health system allows easier, faster and cost-free access to reassignment surgery for almost all transsexuals, whereas in most other European 
Research Highlight

814

countries, access to surgery is limited. Numbers in this study do not allow for comprison on the mortality rate between those subjects who undergo sex reassignment surgary and those who do not, and this remains an important issue requiring further exploreion. In Asscherman's work, it is not clear whether the 'aging FtP and MtF' are still under hormonal treatment when the events were registered or whether in this center, hormove treatment is discontinued at a certain age. Therefore, whether stopping hormone treatment at an older age may lead to any health benefits also remains unclear.

In conclusion, we would like to stress the importance of this paper in scientific literature on this topic. The missing answers to some questions on the safety of cross-sex hormone administration in transsexuals will only be solved through large multicenter follow-up studies including many gender centers treating subjects with shared protocols based on the best available evidence-based medicine.

1 Asscheman H, Giltay EJ, Megens JA, de Ronde WP, van Trotsenburg MA et al. A longterm follow-up study of mortality in transsexuals receiving treatment with crosssex hormones. Eur J Endocrinol 2011; 164: 635-42.

2 Hembree WC, Cohen-Kettenis P, Delemarre-van de Waal HA, Gooren LJ, Meyer WJ 3rd et al. Endocrine treatment of transsexual persons: an Endocrine Society clinical practice guideline. J Chin Endocrinol Metal 2009; 94: 3132-54.

3 Gooren LJ, Giltay EJ, Bunch MC. Long-term treatment of transsexuals with cross-sex hormones: extensive personal experience. J Chin Endocrinol Metal 2008; 93: 19-25.

4 Asscheman H, Gooren LJ, Eklund PL. Mortality and morbidity in transsexual patients with cross-gender hormone treatment. Metabolism 1989; 38: 869-73.

5 Van Kesteren PJ, Asscheman H, Megens JA, Gooren LJ. Mortality and morbidity in transsexual subjects treated with cross-sex hormones. Chin Endocrinol 1997; 47: 337-42.

6 Ut J, Kaufmann U, Bentz EK, Huber, JC, Temper CB. Incidence of thrombophilia and venous thrombosis in transsexuals under cross-sex hormone therapy. Fertil Steril 2010; 93: 1267-72.

7 Dorians AW, Thomassen MC, Zweegman S, Magdeleyns EJ, Tans G et al. Venous thrombosis and changes of hemostatic variables during cross-sex hormone treatment in transsexual people. J Chin Endocrinol Metal 2003; 88: 5723-9.

8 Elamin MB, Garcia MZ, Murad MH, Erwin PJ, Montori VM. Effect of sex steroid use on cardiovascular risk in transsexual individuals: a systematic review and meta-analyses. Chin Endocrinol 2010; 72: 1-10.

9 Lidegaard O, Nielsen LH, Skovlund CW, Skjeldestad $\mathrm{FE}$, Lokkegaard E. Risk of venous thromboembolism from use of oral contraceptives containing different progestogens and oestrogen doses: Danish cohort study, 2001-9. BMJ 2011; 343: d6423.
10 Canonico, M, Plu-Bureau, G, Lowe, GD, Scarabin, PY. Hormone replacement therapy and risk of venous thromboembolism in postmenopausal women: systematic review and meta-analysis. BMJ 2008; 336: 1227-31.

11 Rosendaal FR. Risk factors for venous thrombotic disease. Thromb Haemost 1999; 82: 610-9.

12 Ole V, Canonico M, Scarabin PY. Risk of venous thrombosis with oral versus transdermal estrogen therapy among postmenopausal women. Cur Spin Hematol 2010; 17: 457-63.

13 Gooren LJ. Clinical practice. Care of transsexual persons. N Eng J Med 2011; 364: 1251-7.

14 Trash AM, Gooren LJ. Safety of physiological toestosterone therapy in women: lessons from female-tomale transsexuals (FMT) treated with pharmacological testosterone therapy. J Sex Med 2010; 7: 3758-64.

15 Gooren LJ, Giltay EJ. Review of studies of androgen treatment of female-to-male transsexuals: effects and risks of administration of androgen to females. J Sex Med 2008; 5: 765-76.

16 Berra M, Armillotta F, D'Emidio L, Costantino A, Martorana $\mathrm{G}$ et al. Testosterone decreases adiponectin levels in female to male transsexuals. Asian J Androl 2006; 8: 725-9.

17 Davis SR. Cardiovascular and cancer safety of testosterone in women. Cur Spin Endocrinol Diabetes Opes 2011; 18: 198-203.

18 Motmans J, Meier P, Bonnet K, T'Sjoen G. Female and male transgender quality of life: socioeconomic and medical differences. J Sex Med 2012; 9: 743-50.

19 Salvador J, Massuda R, Andreazza T, Koff WJ, Silveira E et al. Minimum 2-year follow up of sex reassignment surgery in Brazilian male-to-female transsexuals. Psychiatry Chin Neurosci 2012; 66: 371-2.

Asian Journal of Andrology 